\title{
Association between Wnt inhibitory factor-1 expression levels in articular cartilage and the disease severity of patients with osteoarthritis of the knee
}

\author{
SHU-GUANG GAO ${ }^{1,2^{*}}$, CHAO ZENG $^{1 *}$, JUN-JIE LIU $^{1}$, JIAN TIAN $^{1}$, CHAO CHENG $^{1}$, \\ FANG-JIE ZHANG ${ }^{1}$, YI-LIN XIONG ${ }^{1}$, DING PAN ${ }^{1}$, YONG-BING XIAO ${ }^{1}$ and GUANG-HUA LEI ${ }^{1,2}$ \\ ${ }^{1}$ Department of Orthopedics; ${ }^{2}$ Orthopedics Institute of Central South University; ${ }^{3}$ Department of Emergency, \\ Xiangya Hospital, Central South University, Changsha, Hunan 410008; ${ }^{4}$ Department of Orthopedics, \\ Shenzhen Shekou People's Hospital, Shenzhen, Guangdong 518067; ${ }^{5}$ Department of Orthopedics, \\ Yiyang Central Hospital, Yiyang, Hunan 413000, P.R. China
}

Received November 20, 2014; Accepted January 11, 2016

DOI: 10.3892/etm.2016.3049

\begin{abstract}
Wnt inhibitory factor (WIF)-1 is a potent extracellular Wnt antagonist which may be used as a potential molecular therapy for the treatment of inflammatory and autoimmune diseases. Although previous studies have demonstrated that WIF-1 has a protective role in experimental studies of arthritis, its role in the various disease grades of osteoarthritis (OA) remains unclear. A total of 40 patients with various stages of primary OA of the knee and 10 control subjects were enrolled in the present study. Articular cartilage specimens were harvested from subjects following total knee arthroplasty or knee above amputation. Disease severity was determined according to Modified Mankin score and cartilage tissues were ascribed to four groups: Normal, mild, moderate and severe lesions. WIF-1 expression levels in articular cartilage were measured using immunohistochemical techniques. WIF-1 expression levels were detected in all cartilage tissues. As compared with the controls, patients with OA exhibited significantly decreased WIF-1 expression levels in the articular cartilage $(0.19 \pm 0.05$ vs $0.26 \pm 0.04 ; \mathrm{P}<0.01)$. Furthermore, articular cartilage WIF-1 expression levels in the moderate and severe lesion groups were significantly reduced, as compared with the controls $(\mathrm{P}<0.01)$ and mild lesion group $(\mathrm{P}<0.05)$. Subsequent analysis demonstrated that articular cartilage WIF-1 expression levels were negatively correlated with the severity of disease $(r=-0.896$, $\mathrm{P}<0.001)$. In conclusion, the results of the present study suggested that WIF-1 expression levels in articular cartilage
\end{abstract}

Correspondence to: Dr Guang-Hua Lei, Department of Orthopedics, Xiangya Hospital, Central South University, 87 Xiangya Road, Changsha, Hunan 410008, P.R. China

E-mail: lgh9640@gmail.com

${ }^{*}$ Contributed equally

Key words: Wnt inhibitory factor-1, articular cartilage, osteoarthritis may be negatively associated with progressive joint damage in patients with OA of the knee; therefore, WIF-1 expression may be a potential indictor for monitoring OA disease severity.

\section{Introduction}

Osteoarthritis (OA) is a common chronic joint disease, which is characterized by cartilage degradation, synovitis, subchondral bone sclerosis and osteophyte formation (1). Worldwide, the World Health Organization estimates that $\sim 10 \%$ of men and $18 \%$ of women aged $>60$ years have symptomatic OA, $80 \%$ of which suffer from movement limitations (2). OA imposes a substantial burden of disease at the global, regional and individual levels, and this is likely to increase with time due to an aging world population with increasing rates of obesity (3-5). Although previous clinical studies on OA have been performed, the etiology of this disease is yet to be elucidated (6). Several biochemical and biomechanical factors are attributable to the pathogenesis of OA.

Wnt inhibitory factor-1 (WIF-1) is a secreted protein that antagonizes Wnt signaling (7). WIF-1 was initially detected in the human retina and highly conserved homologs have previously been described in various vertebrates (8). WIF-1 mRNA expression has been detected in numerous murine and human tissues, and WIF-1 expression was demonstrated to be most abundant in the brain, lung, retina and cartilage (8-12). Surmann-Schmitt et al (11) demonstrated that WIF-1 is capable of disrupting the CTGF-dependent induction of Acan and COL2A1 gene expression in primary murine chondrocytes. These results indicated that WIF-1 may be a multifunctional modulator of signalling pathways in the cartilage compartment. Stock et al (12) demonstrated that WIF-1 promoted local bone erosion and systemic bone loss whilst protecting against arthritis-triggered cartilage degradation, indicating that WIF-1 may function to rescue the articular surface from excessive Wnt signals.

To the best of our knowledge, there have been no detailed studies investigating WIF-1 expression levels in the articular cartilage of patients with various stages of OA of the knee. 
Whether WIF-1 expression levels in articular cartilage are associated with the disease severity of OA of the knee remains unclear. We hypothesized that WIF-1 in articular cartilage may be associated with disease severity in patients with OA of the knee. The aim of the present study was to investigate the expression levels of WIF-1 in the articular cartilage of patients with primary OA of the knee, and identify the possible correlations with the Modified Mankin score (MMS) of OA of the knee, which may serve as a useful tool for indicating the disease severity and progression of OA of the knee.

\section{Materials and methods}

Patients and preparation of samples. The protocol of the present study was approved by the Human Ethics Committee of Xiangya Hospital (Changsha, China). According to the criteria outlined by the American College of Rheumatology (13), 40 patients aged between 48 and 79 years with no history of any form of secondary OA or inflammatory joint diseases, including rheumatoid arthritis, were eligible for enrollment in the present study. A total of 40 human articular cartilage samples were harvested from the lateral and medial sides of the tibia plateau of the 40 knees of these patients who had undergone total knee arthroplasty (TKA). Radiographs of the knee were captured and evaluated using the Kellgren-Lawrence grading scale (14). Control experiments were performed on 10 samples which were harvested from the disease-free cartilage of patients who had undergone above knee amputation due to severe trauma. Control subjects had no history of secondary OA, knee injury, intra-articular fracture, OA in other joints, osteoporosis, rheumatoid arthritis or tuberculous arthritis, and had not received steroid injection within the preceeding 3 months. Control subjects were matched according to age, gender and body mass index (BMI) with the OA group. Causes of amputation included: Femoral fractures (20\%), open tibial-fibular fractures (50\%) and multiple fractures to the lower leg (30\%). All fractures were complicated with vascular disruptions or destructive soft tissue injuries.

Histology. Biopsies of cartilage and bone were obtained from the lateral and medial sides of the tibia plateau including the loading zone and the margin zone whenever possible. All cartilage samples underwent decalcification with $15 \%$ ethylenediaminetetraacetic acid solution (Beyotime Institute of Biotechnology, Haimen, China) for 3 weeks. Specimens, which were $1.0 \mathrm{~cm}$-thick with a cartilage surface of $\sim 2.0 \times 0.5 \mathrm{~cm}$, were incubated in freshly prepared paraformaldehyde (Tianjin Kemiou Chemical Reagent Co., Ltd., Tianjin, China) prior to dehydration with a graded concentration of ethanol and xylene and paraffin embedding (all Sigma-Aldrich, St. Louis, MO, USA). Subsequently, sagittal $5-\mu \mathrm{m}$ thick sections were cut and stained with hematoxylin and eosin and safranin-O fast green (both Sigma-Aldrich). The severity of cartilage damage was assessed according to the MMS (15): Normal (MMS, $0-1 ; n=10$ ), mild lesions (MMS, 2-5; $n=9$ ), moderate lesions (MMS, 6-9; $n=14$ ) and severe lesions (MMS, 10-14; $\mathrm{n}=15$ ). Two samples were excluded from the present study as the specimens were destroyed.
Immunohistochemistry. Paraffin-fixed samples were deparaffinized in xylene and ethanol prior to rehydration in water. Subsequently, the sections were incubated with trypsin (Beyotime Institute of Biotechnology) for $30 \mathrm{~min}$ at $37^{\circ} \mathrm{C}$ and incubated with citrate sodium (Sigma-Aldrich) in a water bath for $10 \mathrm{~min}$. Following washing with phosphate-buffered saline (PBS), sections were blocked with $0.5 \%$ bovine serum albumin (diluted in PBS; both Sigma-Aldrich) for $30 \mathrm{~min}$ at room temperature. The sections were subsequently incubated with diluted WIF-1 antibody (1:100; CB52917240 Beijing Biosynthesis Biotechnology Co., Ltd., Beijing, China) for $1 \mathrm{~h}$. Following rinsing with PBS, the sections were incubated with horseradish peroxidase/Fab polymer conjugate (Zymed Picure-Plus kit; Thermo Fisher Scientific, Inc., Waltham, MA, USA) for $30 \mathrm{~min}$. Finally, the sections were incubated with 3,3'-diaminobenzidine (Sigma-Aldrich) for $5 \mathrm{~min}$ in order to develop the signals. A negative control was included by omitting the primary antibody. All the sections were examined by an independent pathologist who was unaware of the clinical characteristics of the samples included in the present study. All the sections were examined using a Motic BA210 microscope (Motic Instruments, Xiamen, China) at x100 magnification. The relative WIF-1 distribution of cartilage tissue was visualized and quantified using optical density (OD) values $(16,17)$. Positive WIF-1 immunostaining was defined as detectable immunoreactivity in the perinuclear and/or other cytoplasmic regions in chondrocytes. Semiquantitative assessment of the average optical density of WIF-1 expression levels was performed on scanned autoradiograms using MIAS-4400 ImageJ (National Institutes of Health, Bethesda, MA, USA) (1). All densities were normalized against PBS and the experiment was repeated in triplicate. The coefficient of variation (CV) of WIF-1 expression in articular cartilage was $<2 \%$.

Statistical analysis. Statistical analyses were performed using SPSS software 13.0 (SPSS, Inc., Chicago, IL, USA). One-way analysis of variance was performed in the present study in order to determine between-group differences. Associations between WIF-1 expression levels in the articular cartilage, as determined by OD values, and the MMSs of OA were analyzed using Pearson's correlation analysis. Data were expressed as the mean \pm standard deviation. $\mathrm{P}<0.05$ was considered to indicate a statistically significant difference.

\section{Results}

Patient characteristics. A total of 48 knee samples were analyzed from 38 patients with OA of the knee and 10 control subjects. Baseline clinical characteristics of both groups are presented in Table I. No significant differences were detected in age, gender and BMI indices between the two groups. Kellgren-Lawrence radiological scoring confirmed that the majority of patients with OA of the knee presented with severe OA, which was appropriate for total knee replacement (Table II).

WIF-1 expression levels. MMSs of each group are shown in Table III. WIF-1 expression levels were detected in the articular cartilage of all four groups. In both the control and mild lesion groups, WIF-1 expression was predominantly detected in the 
Table I. Characteristics of patients with osteoarthritis of the knee $(n=38)$ and control subjects $(n=10)$.

\begin{tabular}{lccc}
\hline Parameter & Patients & Controls & P-value \\
\hline Age (years) & $62.0 \pm 13.6$ & $56.2 \pm 18.9$ & 0.66 \\
Gender $(\%$ female $)$ & 45 & 40 & 0.32 \\
BMI $\left(\mathrm{kg} / \mathrm{m}^{2}\right)$ & $28.08 \pm 0.49$ & $26.34 \pm 0.56$ & 0.13 \\
\hline
\end{tabular}

Data are presented as the mean \pm standard deviation. BMI, body mass index.

Table II. Kellgren-Lawrence radiological scores in patients with osteoarthritis of the knee $(n=40)$.

Kellgren-Lawrence score Patients (n)

Grade 1 0

Grade 2

0

Grade 3

9

Grade 4

Table III. Modified Mankin scores in the various groups.

\begin{tabular}{lcc}
\hline Group & Samples (n) & Modified Mankin score \\
\hline Normal & 10 & $0.60 \pm 0.52$ \\
Mild lesions & 9 & $3.11 \pm 1.05$ \\
Moderate lesions & 14 & $7.21 \pm 1.12$ \\
Severe lesions & 15 & $12.07 \pm 1.33$ \\
\hline
\end{tabular}

Data are presented as the mean \pm standard deviation.

superficial layers of the articular cartilage (Fig. 1); whereas faint staining was detected in the territorial matrix of the deep zone of articular cartilage in the moderate and severe lesions (Fig. 1).

$O D$ values. Average OD values of WIF-1 in the articular cartilage was $0.26 \pm 0.04$ in the control patients, $0.19 \pm 0.05$ in patients with $\mathrm{OA}$ of the knee and $0.24 \pm 0.03,0.19 \pm 0.04$ and $0.16 \pm 0.04$ in the mild, moderate and severe lesions, respectively. Patients with OA demonstrated significantly reduced WIF-1 expression levels in the articular cartilage, as compared to the controls $(\mathrm{P}<0.01)$ (Fig. 2). Furthermore, WIF-1 expression levels in the moderate and severe lesions of articular cartilage were significantly reduced, as compared with the control $(\mathrm{P}<0.01)$ and mild lesion $(\mathrm{P}<0.05)$ groups (Fig. 2). The MMSs ( $\mathrm{r}=-0.896$; $\mathrm{P}<0.001)$ indicate that WIF-1 expression in articular cartilage was negatively correlated with severity of disease (Fig. 3).

\section{Discussion}

To the best of our knowledge, the present study is the first to investigate the WIF-1 expression levels of articular cartilage

in patients with various stages of OA of the knee and its correlation with OA disease severity. The results of the present study demonstrated that WIF-1 expression is predominantly detected in the superficial layers of articular cartilage. These results are consistent with previous studies which have demonstrated that WIF-1 is highly expressed in the superficial layers of epiphyseal cartilage in bone and tendons $(11,12,18)$. Since WIF-1 is predominantly expressed in articular cartilage, and in bone to a lesser extent $(12,18)$, the present study aimed to investigate whether WIF-1 is upregulated in patients with OA. Notably, the present study demonstrated a marked reduction in WIF-1 expression levels in the articular cartilage of patients with $\mathrm{OA}$ of the knee, as compared with the controls.

Cartilage damage is one of the predominant pathological changes detected in patients with OA. According to the MMS, the results of the present study demonstrated that WIF-1 expression levels in articular cartilage were negatively correlated with the severity of disease. These results suggested that the loss of WIF-1 expression may be an early event in the OA disease progress and indicated that WIF-1 may be associated with the pathogenesis of OA. A previous study demonstrated specific binding of WIF-1 to Wnt3a, Wnt4, Wnt5a, Wnt7a, Wnt9a and Wnt11 (18). Furthermore, it has been demonstrated that WIF-1 is capable of blocking Wnt3a-mediated activation of the canonical Wnt signalling pathway, which is associated with cartilage degeneration (18). The results of another previous study demonstrated that WIF-1 was capable of attenuating the CTGF-dependent induction of cartilage matrix gene expression, including aggrecan and COL2A1, in primary murine chondrocytes (11). Therefore, this mechanism, which is mediated by WIF-1, may have a key role in OA.

Stock et al (12) demonstrated that WIF-1 expression levels were repressed by tumor necrosis factor (TNF) $\alpha$ in chondrocytes and osteoblasts, and were downregulated in inflammatory arthritis. WIF-1 deficiency partially protected TNF-transgenic mice against bone loss and reduced arthritis-related increases in osteoclast numbers. Enhanced cartilage destruction was detected during WIF-1 deficiency and systemic overexpression of WIF-1 was demonstrated to partially protect against arthritis-related cartilage loss, which suggested that WIF-1 may have a protective role in cartilage destruction in patients with arthritis. Furthermore, in chondrocytes, TNF $\alpha$ induced canonical Wnt signaling, which was successfully blocked by WIF-1, indicating that TNF $\alpha$ and WIF-1 may have a direct effect on Wnt signaling in this system. These data suggested that WIF-1 may be associated with the fine-tuning of cartilage and bone turnover, thus promoting the balance between cartilage and bone anabolism.

Aberrant methylation of regulatory regions that silence the transcription of genes has been postulated to be a mechanism for the inactivation of tumor suppressor genes in human cancer (19-22). Mazieres et al (23) demonstrated that methylation silencing of WIF-1 was a common mechanism of aberrant activation of the Wnt signaling pathway in lung cancer pathogenesis. Furthermore, Urakami et al (21) previously demonstrated that, independent of histological grade or stage, bladder tumor samples exhibited decreased WIF-1 expression levels and enhanced WIF-1 promoter methylation, as compared with normal bladder mucosa samples. Therefore, 

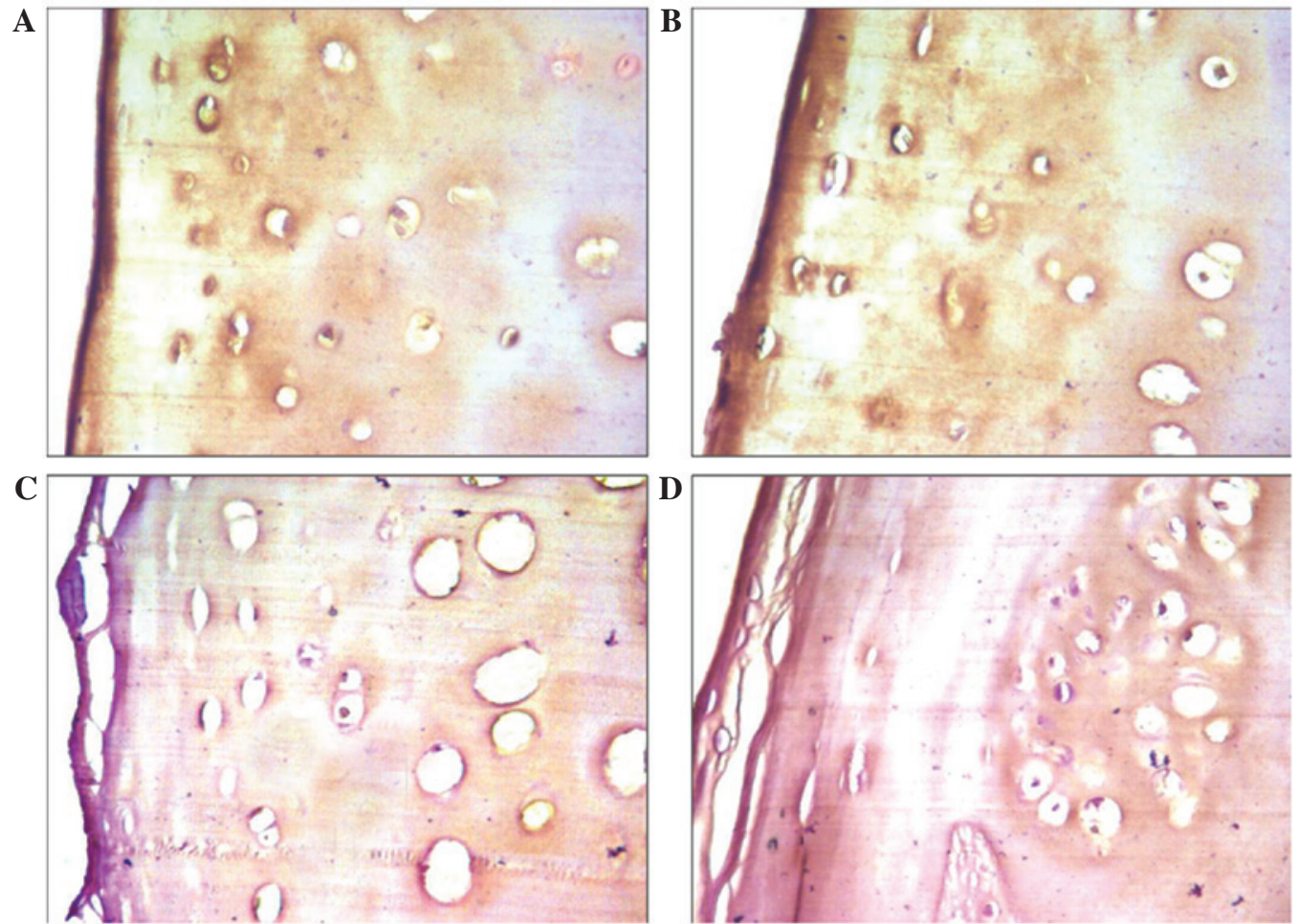

Figure 1. WIF-1 immunohistochemical staining in (A) normal cartilage and cartilage with (B) mild, (C) moderate and (D) severe lesions.

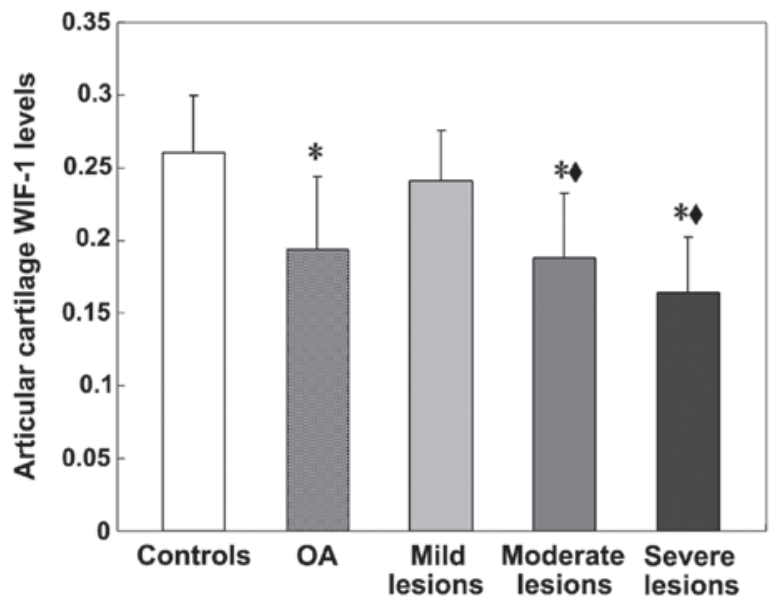

Figure 2. WIF-1 expression levels in the articular cartilage of the control group $(n=10)$ and patients with osteoarthritis of the knee $(n=38)$, whom were characterized according to the severity of cartilage damage into mild $(n=9)$, moderate $(\mathrm{n}=15)$ and severe $(\mathrm{n}=14)$ lesion groups, using the Modified Mankin score. ${ }^{*} \mathrm{P}<0.01$ vs. control group; ${ }^{\mathrm{P}} \mathrm{P}<0.05$ vs. mild lesions group.

it is possible that dysregulated WIF-1 expression, induced by promoter hypermethylation, may activate the Wnt/ $\beta$-catenin signaling pathway via the nuclear translocation of $\beta$-catenin in the pathogenesis of OA.

The present study had various limitations. Firstly, the present study had a small sample size and was a single-center study. Secondly, only patients with OA of the knee who attended Xiangya Hospital were enrolled in the study. Thirdly, the associations between WIF-1 expression levels in the serum, synovial fluid and articular cartilage, which were determined via radiographic grading of OA, were not analyzed. Therefore, in order to determine whether WIF-1 expression levels are capable of

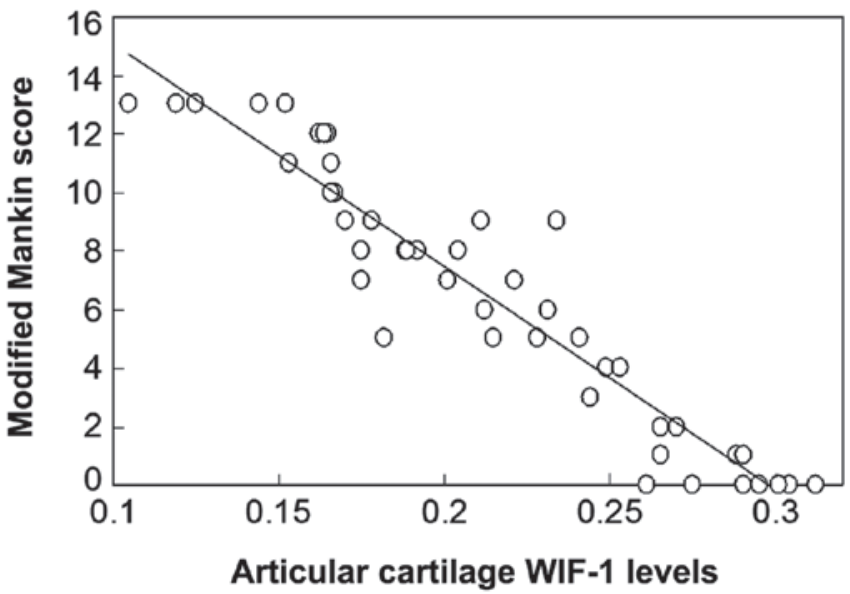

Figure 3. Articular cartilage WIF-1 expression levels are negatively correlated with the severity of disease $(r=-0.896, \mathrm{P}<0.001)$. Osteoarthritic changes were histomorphologically classified using the Modified Mankin score.

predicting clinical prognosis and outcomes in patients with $\mathrm{OA}$ of the knee, a cross-sectional study is required.

In conclusion, the results of the present study demonstrated that patients OA of the knee exhibited reduced WIF-1 expression levels, as compared with healthy control subjects. Furthermore, WIF-1 expression levels in articular cartilage were demonstrated to be negatively associated with the MMS, indicating that WIF-1 expression may be a potential indictor of disease severity of OA of the knee. Further investigations are being performed by the authors of the present study in order to define WIF-1 expression levels in the synovial fluid or serum, the signaling events induced by WIF-1 and the potential utility 
of WIF-1 as a therapeutic target to inhibit the progression of OA. Measurements of WIF-1 expression levels in the synovial fluid or serum may have predictive value for the progression of OA of the knee. Subsequent longitudinal studies may further elucidate the therapeutic value of WIF-1 as a potential marker to monitor the disease severity and progression of OA of the knee.

\section{Acknowledgements}

The present study was supported by the National Natural Science Foundation of China (grant nos. 81201420 and 81272034), the Provincial Science Foundation of Hunan (grant no. 14JJ3032), the Scientific Research Project of the Development and Reform Commission of Hunan Province (grant no. 20131199), the Scientific Research Project of Science and Technology Office of Hunan Province (grant no. 2013SK2018), the Doctoral Scientific Fund Project of the Ministry of Education of China (grant no. 20120162110036) and the Clinical Research Project of Xingya Hospital (grant no 2015101).

\section{References}

1. Arden NK and Leyland KM: Osteoarthritis year 2013 in review: Clinical. Osteoarthritis Cartilage 21: 1409-1413, 2013.

2. Gao SG, Li KH, Zeng KB, Tu M, Xu M and Lei GH: Elevated osteopontin level of synovial fluid and articular cartilage is associated with disease severity in knee osteoarthritis patients. Osteoarthritis Cartilage 18: 82-87, 2010.

3. Lawrence RC, Felson DT, Helmick CG, Arnold LM, Choi H, Deyo RA, Gabriel S, Hirsch R, Hochberg MC, Hunder GG, et al: Estimates of the prevalence of arthritis and other rheumatic conditions in the United States. Part II. Arthritis Rheum 58: 26-35, 2008.

4. Liu M and Hu C: Association of MIF in serum and synovial fluid with severity of knee osteoarthritis. Clin Biochem 45: 737-739, 2012.

5. Holt HL, Katz JN, Reichmann WM, Gerlovin H, Wright EA, Hunter DJ, Jordan JM, Kessler CL and Losina E: Forecasting the burden of advanced knee osteoarthritis over a 10 -year period in a cohort of 60-64 year-old US adults. Osteoarthritis Cartilage 19: 44-50, 2011.

6. Bay-Jensen AC, Reker D, Kjelgaard-Petersen CF, Mobasheri A, Karsdal MA, Ladel C, Henrotin $Y$ and Thudium CS: Osteoarthritis year in review 2015: soluble biomarkers and the BIPED criteria. Osteoarthritis Cartilage 24: 9-20, 2016

7. Ai L, Tao Q, Zhong S, Fields CR, Kim WJ, Lee MW, Cui Y, Brown KD and Robertson KD: Inactivation of Wnt inhibitory factor-1 (WIF1) expression by epigenetic silencing is a common event in breast cancer. Carcinogenesis 27: 1341-1348, 2006.

8. Hsieh JC, Kodjabachian L, Rebbert ML, Rattner A, Smallwood PM, Samos CH, Nusse R, Dawid IB and Nathans J: A new secreted protein that binds to Wnt proteins and inhibits their activities. Nature 398: 431-436, 1999.

9. Hu YA, Gu X, Liu J, Yang Y, Yan Y and Zhao C: Expression pattern of Wnt inhibitor factor 1(Wif1) during the development in mouse CNS. Gene Expr Patterns 8: 515-522, 2008.
10. Hunter DD, Zhang M, Ferguson JW, Koch M and Brunken WJ: The extracellular matrix component WIF-1 is expressed during and can modulate, retinal development. Mol Cell Neurosci 27: 477-488, 2004

11. Surmann-Schmitt C, Sasaki T, Hattori T, Eitzinger N, Schett G, von der Mark K and Stock M: The Wnt antagonist Wif-1 interacts with CTGF and inhibits CTGF activity. J Cell Physiol 227: 2207-2216, 2012.

12. Stock M, Böhm C, Scholtysek C, Englbrecht M, Fürnrohr BG, Klinger P, Gelse K, Gayetskyy S, Engelke K, Billmeier U, et al: Wnt inhibitory factor 1 deficiency uncouples cartilage and bone destruction in tumor necrosis factor $\alpha$-mediated experimental arthritis. Arthritis Rheum 65: 2310-2322, 2013.

13. Altman R, Asch E, Bloch D, Bole G, Borenstein D, Brandt K, Christy W, Cooke TD, Greenwald R and Hochberg M: Development of criteria for the classification and reporting of osteoarthritis: Classification of osteoarthritis of the knee Diagnostic and Therapeutic Criteria of the American Rheumatism Association. Arthritis Rheum 29: 1039-1049, 1986.

14. Kellgren JH and Lawrence JS: Radiological assessment of osteo-arthrosis. Ann Rheum Dis 16: 494-502, 1957.

15. van der Sluijs JA, Geesink RG, van der Linden AJ, Bulstra SK, Kuyer R and Drukker J: The reliability of the Mankin score for osteoarthritis. J Orthop Res 10: 58-61, 1992.

16. Jiang W, Gao SG, Chen XG, Xu XC, Xu M, Luo W, Tu M, Zhang FJ, Zeng C and Lei GH: Expression of synovial fluid and articular cartilage VIP in human osteoarthritic knee: A new indicator of disease severity? Clin Biochem 45: 1607-1612, 2012.

17. Zhang FJ, Luo W, Gao SG, Su DZ, Li YS, Zeng C and Lei GH: Expression of CD44 in articular cartilage is associated with disease severity in knee osteoarthritis. Mod Rheumatol 23: 1186-1191, 2013

18. Surmann-Schmitt C, Widmann N, Dietz U, Saeger B, Eitzinger N, Nakamura Y, Rattel M, Latham R, Hartmann C, von der Mark $\mathrm{H}$, et al: Wif- 1 is expressed at cartilage-mesenchyme interfaces and impedes Wnt3a-mediated inhibition of chondrogenesis. J Cell Sci 122: 3627-3637, 2009.

19. Herman JG and Baylin SB: Gene silencing in cancer in association with promoter hypermethylation. N Engl J Med 349: 2042-2054, 2003.

20. Jones PA and Baylin SB: The fundamental role of epigenetic events in cancer. Nat Rev Genet 3: 415-428, 2002.

21. Urakami S, Shiina H, Enokida H, Kawakami T, Tokizane T, Ogishima T, Tanaka Y, Li LC, Ribeiro-Filho LA, Terashima M, et al: Epigenetic inactivation of Wnt inhibitory factor-1 plays an important role in bladder cancer through aberrant canonical Wnt/beta-catenin signaling pathway. Clin Cancer Res 12: 383-391, 2006.

22. Yang Z, Wang Y, Fang J, Chen F, Liu J, Wu J and Wang Y: Expression and aberrant promoter methylation of Wnt inhibitory factor-1 in human astrocytomas. J Exp Clin Cancer Res 29: 26, 2010.

23. Mazieres J, He B, You L, Xu Z, Lee AY, Mikami I, Reguart N, Rosell R, McCormick F and Jablons DM: Wnt inhibitory factor-1 is silenced by promoter hypermethylation in human lung cancer. Cancer Res 64: 4717-4720, 2004. 